\title{
Public Transport Operations After Lockdown: How to Make It Happen?
}

\author{
Hemant K. Suman ${ }^{1} \cdot$ Amit Agarwal' ${ }^{2}$ (1) Nomesh B. Bolia ${ }^{3}$ \\ Received: 27 April 2020 / Revised: 26 May 2020 / Accepted: 30 May 2020 / Published online: 12 June 2020 \\ (C) Indian National Academy of Engineering 2020
}

\begin{abstract}
Lockdown in cities across the globe has imposed severe travel restrictions to limit the spread of Coronavirus disease. The travel behavior and operations will not be the same as before due to requirements such as physical (social) distancing. This study analyzes the resulting shortage in supply of public transport (buses) that will likely widen the existing gap between demand and supply. In this work, system optimization models are developed to efficiently reallocate the bus fleet to routes for different levels of physical distancing gaps and travel demand. The proposed models are applied to a real-life network of 34 bus routes of Delhi, considering three types of scenarios: current, practical, and ideal. In the practical scenarios, the additional, idling bus fleets can be allocated to the routes efficiently while maintaining physical distancing. The results show that the Business-as-Usual (BAU) scenario involving the current allocation approach will make it impossible to use public buses even if the bare minimum physical distancing has to be maintained. Further, the models proposed in the study significantly improve the key performance indicators for all scenarios.
\end{abstract}

Keywords Public transport · Coronavirus · Decision support · Mathematical modeling $\cdot$ Post-COVID-19 $\cdot$ Lockdown

\section{Introduction}

\section{Context}

The outbreak of novel Coronavirus (formally, SARSnCoV-2 or nCov-19) has brought almost every other city to a standstill. This is a respiratory virus that primarily spreads through close contact with an infected person and/or fomites (e.g., handrails, doorknob, cloths, etc.). To minimize the contact with each other or any common surface, extreme measures such as lockdown of cities (with very limited mobility for essential services), prohibiting mass gathering (e.g., in schools, colleges, institutes, religious places, etc.) are recommended (Müller et al. 2020; Fang et al. 2020) and observed all across the globe. Similar to this but for a far less severe situation, it has been shown that holiday

Amit Agarwal

amitfce@iitr.ac.in

1 Department of Transport Engineering and Logistics, Pontificia Universidad Catòlica de Chile, Santiago, Chile

2 Department of Civil Engineering, Indian Institute of Technology Roorkee, Roorkee, India

3 Department of Mechanical Engineering, Indian Institute of Technology Delhi, New Delhi, India traveling may lead to a potential second peak of an influenza pandemic (Shi et al. 2010). The public transport system is considered at high risk due to higher passenger density, mainly in peak hours, higher chances of getting the infection from common surfaces, and difficulty in the screening of the individuals. Therefore, public transport systems have ground to a halt. Specifically, in India, the first case was reported on Jan 20, 2020 (WHO 2020a), the number of cases rapidly crossed the 21,000 mark on Apr 23, 2020 (WHO 2020b), and a nation-wide lockdown was initiated on Mar 25, 2020.

As the situation improves, the lockdown will be relaxed, public movement will begin, and services will gradually resume. However, it is a long road before the situation normalizes. Due to the fear of getting infected, it is likely that many people will continue to work from home, use private vehicles (e.g., car, motorbike) or an active mode of transport (e.g., walk, bicycle) for shorter distances. However, public transport is one of the most important modes of mobility that is sustainable and serves people at a large scale. Specifically, low-income travelers walk/cycle or use public transport extensively (Rangwala et al. 2014; Tiwari et al. 2016), i.e., it is unlikely for them to travel for long-distance trips in the absence of public transport. At the same time, in the prevailing circumstances, public transport users are at high risk, which will stymie its operation. Thus, in order to avoid 
the risk of getting infected, it is highly likely and desirable that for some time, maintaining physical (social) distancing becomes a requirement for all public vehicles and seating, standing spaces are clearly marked. To achieve the required physical distancing in the already short-in-supply transit fleet (Agrawal et al. 2020), this study attempts to evaluate the requirement of an operational fleet of public transport immediately after the relaxation in the lockdown and better prepare for the situation. A better preparation of the public transport operators will also avoid the overcrowding at the platforms. In this study, 'post-COVID-19' refers to the situation when the lockdown is relaxed fully or partially, and public transport is opened for the public.

\section{Novelty of the Work}

This work first attempts to identify and address the gap between the demand and supply of public transport for different levels of physical distancing and levels of travel demand. Further, an optimization approach is proposed to bridge the gap between the derived travel demand and available fleet size by effective utilization of additional buses along with the existing ones and make public transport operational for a given scenario. In order to avoid the overestimation of additional buses required to serve the travel demand, reduction in travel demand is estimated using the geometry of the line, possibility of travel using alternate route/transit mode, and production centers.

The proposed optimization approach is a set of Mixed Integer Linear Programming (MILP) optimization models. In this work, three MILP models that respectively maximize the total passenger hours, travel demand, and the number of routes opened are developed by efficiently reallocating the existing as well as additional buses to the routes. These models are inspired by and an extension of the work done by Suman and Bolia (2019). Apart from optimal allocation of buses, the study identifies the additional bus fleet required to meet the travel demand (Suman and Bolia 2019) while maintaining the chosen (policy-mandated) level of physical distancing. The models proposed in this work can be a key aid to policymakers in serving the maximum possible mobility needs of the people in the post-Covid19 world.

\section{Methodology}

\section{Assumptions}

Given the uncertainty associated with the novel Coronavirus, the following assumptions are taken in the present study: (1) the lockdown will be relaxed in different stages; therefore, in the first stage, the inter-state and tourist buses will have the least priority to operate; (2) the govt. has the authority to use the idling inter-state and tourist buses for public transport services; (3) if in operation, all measures will be taken to ensure the screening, safety of passengers as well as the driver and conductor.

\section{Reducing the Travel Demand}

It is likely that the lockdown will be relaxed in a staged manner, and work from home will be recommended for a longer period of time. Further, travelers have a fear of getting infected, which is likely to encourage them to avoid public transport and use private vehicles. Therefore, once the authorities start the operation of public transport, it is expected that the travel demand is likely to be lesser than normal circumstances (prior to COVID-19).

Ideally, to determine the reduction in travel demand, detailed modeling and various data inputs are required. Based on the availability of the data (see "Case Study: Delhi, India"), the following approach, depending primarily on the alternative route/mode of public transport, geometry of transit line, and the number of trip attraction centers, is devised. For the former, the number of transfer stations with other buses/metro lines, the number of metro lines that have three or more consecutive stations in parallel to the bus line (route) are counted using the map services. These numbers indicate the likelihood of serving the trip using other transit lines. The geometry of line represents segregation of the trip purposes, i.e., trips served by a tangential (or cross-town) transit line are lesser peaked than radial and diametric lines and served primarily for educational, social, leisure trips, etc. On the contrary, the radial and diametric lines mostly serve the daily commuters (Vuchic 2004), who have higher priority over other types of trips. Higher number of trip attractions (e.g., shopping malls/street/hubs, tourist destinations, Museums, stadiums, etc.) as well as higher number of multi-modal interchanges (e.g., ISBT, Railway stations, etc.) point towards a greater reduction in travel demand due to non-operation of these attractions/services. The values corresponding to each attribute are identified for each transit route using map services, a weight ${ }^{1}$ is assigned to and normalization done for each attribute. The sum of weighted, normalized attributes for each route is termed as the 'remaining demand multiplier' for that route, and is multiplied with the transit demand between each OD pair of the route to get the reduced demand. ${ }^{2}$ Given the uncertainty in

\footnotetext{
1 The weights are assumed as 0.25 for major attractions, 0.20 for multi-modal interchanges, 0.20 for the geometry of line, 0.15 for the number of metro lines with three consecutive metro stations, and 0.10 each for the number of transfer stations with other bus/metro lines.

${ }^{2}$ Note that each bus line has two routes in both directions. For a few bus lines, the routes in the two directions vary slightly. However, the same multipliers are used in either direction.
} 


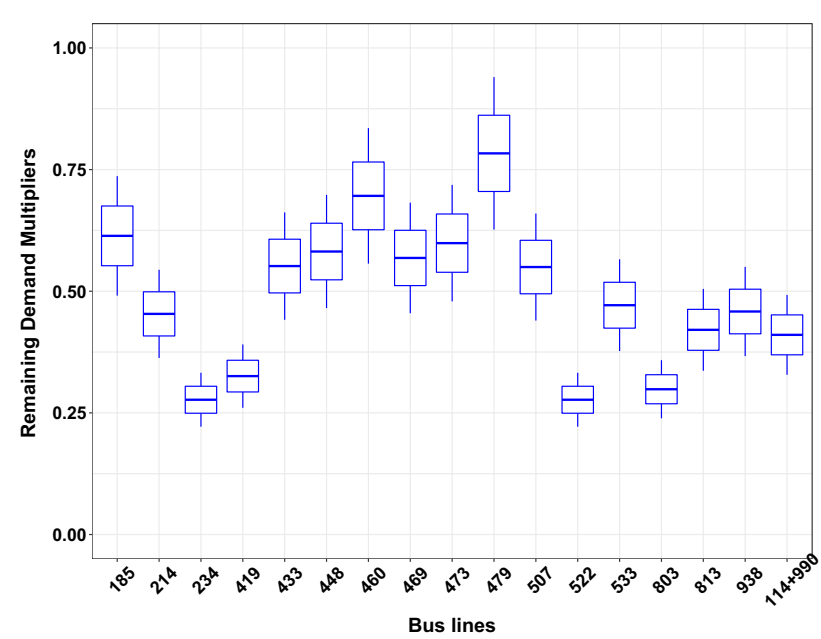

Fig. 1 Remaining demand multipliers for bus lines

the travel demand post-COVID-19, a sensitivity analysis is performed for $\pm 10, \pm 20 \%$ of the remaining demand multipliers. Figure 1 shows the variation of remaining demand multipliers for all bus routes in terms of a box plot. Clearly, for a few routes, the demand is likely to be just above $20 \%$, whereas on the higher side, for route 479 , the demand is likely to remain between 62 and $94 \%$ of the original demand.

\section{Bus Fleet and Capacities}

Under normal circumstances, the required bus fleet in Delhi is not enough to serve the demand; however, a better route allocation can reduce the demand-supply gap (Suman and Bolia 2019). Clearly, the gap between demand and supply will widen significantly to enable physical distancing. As mentioned in "Novelty of the Work", this work proposes a methodology to determine the number of additional buses required to serve the reduced demand constrained by different levels of physical distancing in the buses. The requirement of the additional buses can be fulfilled using the 10,000 buses which have permits under contract carriage, all India tourist, inter-state and stage carrier categories. ${ }^{3}$ During lockdown, all of these buses are idling and unlikely to go in operation together as soon as the lockdown is relaxed. For instance, tourism may remain closed for a few more weeks/months. For the present study, four different categories of buses are assumed to be available, of which two are already in use for public transport. Three different values of distances among the passengers are assumed, and the bus

\footnotetext{
3 As per the data by Transport Department, Govt. of NCT of Delhi (2020), more than 10,000 bus permits are valid between 2015 and 2020, and therefore, the same number is assumed to be valid at any given time.
}

Table 1 Bus category and capacities based on social distancing

\begin{tabular}{llllll}
\hline Bus category & $\begin{array}{l}\text { Bus } \\
\text { length } \\
(\mathrm{m})\end{array}$ & $\begin{array}{l}\text { Seating } \\
\text { capacity }\end{array}$ & \multicolumn{2}{l}{ Social-distancing gap } \\
\cline { 4 - 6 } \cline { 4 - 6 } & 10 & 26 & 23 & 11 & 5 \\
Tourist & 12 & 34 & 29 & 14 & 7 \\
Low-floor & 12 & 40 & 32 & 16 & 8 \\
Semi-low floor & 12 & 52 & 38 & 19 & 9 \\
Inter-state & 12 & $52 \mathrm{~m}$ & \\
\hline
\end{tabular}

capacity is calculated for each (see Table 1). The total capacity of a bus is sum of the standing and seating capacities. For simplicity, in all buses, it is assumed that seats are of dimension $0.45 \mathrm{~m} \times 0.45 \mathrm{~m}$, the center to center distance for each seating row is about $0.6 \mathrm{~m}$. Based on this, the standing capacity is calculated from the length of the bus, i.e., a person can stand in every other row (e.g., for a $10 \mathrm{~m}$ long bus and $1 \mathrm{~m}$ social distancing gap, five persons can stand). Similarly, the seating capacity is half of seating capacity in alternative row derived from the social distancing gap (e.g., for seating capacity of 26 and $1 \mathrm{~m}$ social distancing gap, six persons can be seated). ${ }^{4}$

\section{Mathematical Model}

Since the present study focuses on providing better public transit service through a mathematical program to reallocate the given number of buses to the routes, the planning horizon and total vehicle hours, and therefore the total operating cost will not change significantly across its various solutions. Accordingly, the operating cost is not considered in the objective functions to avoid any complexity in the models. The list of notations used in the models are presented in Table 2 and the objective functions of the three developed models are shown by Eqs. 1-3.

Equation 1 maximizes the total passenger hours for a given fleet (see Eq. 4) while maintaining the assigned physical distancing (see Table 1). Maximizing this objective function is likely to give higher priority to serve longer trips and reduce the shorter trips. This is desirable because active transport (e.g., walk or bicycle) is recommended for shorter trips due to its better ability to maintain distancing and minimize contact. The objective function in Eq. 3 maximizes the number of routes on which the mandated physical distancing can be maintained using the available fleet. However, this will tend to increase the service to the routes with a lesser demand and serve shorter trips. The Eq. 2 maximizes the

\footnotetext{
${ }_{4}$ A schematic of seating arrangement in the bus and, Dos and Don'ts in the public transit vehicles and at the transit stop are shown in the technical report by CRRI (2020).
} 
Table 2 List of notations

\begin{tabular}{lll}
\hline Category & Symbol & Description \\
\hline Sets & $K$ & Set of routes in the network \\
& $S$ & Set of bus stops in the network \\
& $s_{k}$ & Set of bus stops in route $k$, i.e., $s_{k} \subseteq S$ and $n\left(s_{k}\right)$ is the number of stops on route $k$ \\
& $A_{k}$ & Set of arcs connecting the consecutive bus stops of route $k$ \\
Variables & $f_{m}^{k}$ & Set representing the type of buses, where each type of bus $m \in M$ \\
& $\chi_{k}$ & Integer number of type $m$ buses assigned to route $k$ \\
& $\vartheta_{a}^{k}$ & Binary decision variable, equals 1 if minimum social distancing over route $k$ can \\
& & Auxiliary binary variable, equals 1 if minimum social distancing over arc $a$ of \\
Parameters & $D_{i j}^{k}$ & Travel demand between stops $i$ and $j$ over route $k$ \\
& $\alpha_{i}^{k}$ & Cumulative number of commuters alighted from stop 1 to $i$ on route $k$ \\
& $\beta_{i}^{k}$ & Cumulative number of commuters boarded from stop 1 to $i$ on route $k$ \\
& $\tau_{i j}^{k}$ & Travel time between stops $i$ and $j$ over route $k$ \\
& $N_{m}$ & Number of type $m$ buses available in the given planning horizon \\
& $b_{m}$ & Effective capacity of type $m$ buses \\
$\check{T}_{k}$ & Ratio of time of decision horizon to cycle time of the route
\end{tabular}

total demand, which can be served by the given fleet while satisfying the distancing constraints. The Eqs. 1-3 are called as 'optimizing Passenger Hours (PH)', 'optimizing Travel Demand (TD)' and 'optimizing Number of Routes (NR)' respectively (see Fig. 3), and the decision makers can choose whichever better suits their policy priorities.

\section{Models:}

$$
\begin{aligned}
& \underset{\left\{\chi_{k}\right\}}{\operatorname{aaximize}}\left[\sum_{k \in K} \chi_{k}\left(\sum_{i \in S_{k}} \sum_{j>i} D_{i j}^{k} \cdot \tau_{i j}^{k}\right)\right] \\
& \underset{\left\{\chi_{k}\right\}}{\operatorname{Maximize}}\left[\sum_{k \in K} \chi_{k}\left(\sum_{i \in S_{k}} \sum_{j>i} D_{i j}^{k}\right)\right] \\
& \underset{\left\{\chi_{k}\right\}}{\operatorname{Maximize}}\left[\sum_{k \in K} \chi_{k}\right]
\end{aligned}
$$

\section{Subject to:}

$$
\sum_{k \in K} f_{m}^{k} \leq N_{m} \quad \forall m \in M
$$

$$
\vartheta_{a}^{k}\left(\sum_{i \leq a} \beta_{i}^{k}-\sum_{i \leq a} \alpha_{i}^{k}\right) \leq \sum_{m \in M} f_{m}^{k} \cdot \check{T}_{k} \cdot b_{m}, \forall a \in A_{k}, \forall k \in K
$$

$$
\chi_{k} \leq \min _{a \in A_{k}}\left\{\vartheta_{a}^{k}\right\} \quad \forall k \in K
$$

$\vartheta_{a}^{k}, \chi_{k} \in\{0,1\} \quad \forall a \in A_{k} \quad \forall k \in K$

$f_{m}^{k} \geq 0$, integer $\quad \forall m \in M \quad \forall k \in K$

$\chi_{k} \cdot n\left(S_{k}\right) \leq 1+\sum_{a \in A_{k}} \vartheta_{a}^{k} \quad \forall k \in K$

Each of these objective functions are subjected to various constraints represented by Eqs. 4-8. The first constraint (Eq. 4) is fleet size constraint and the number of buses assigned to each route is non-negative integer (see Eq. 8). Further, Eq. 5 ensures the required physical distancing since it represents the bus capacity constraint where $b_{m}$ is a function of the gap required for the mandated distancing and bus category (see Table 1), and $\breve{T}_{k}$ is a parameter to determine the number of trips from the number of buses. ${ }^{5}$ Equation 6 links the binary decision variable $\chi_{k}$ and the auxiliary binary decision variable $\vartheta_{a}^{k}$. Equation 7 ensures that these two decision variables are binary.

The models presented through Eqs. 1-8 are mixed integer non-linear programming (MINLP) in nature. The nonlinearity arises due to Eq. 6 since all other constraints and the three objective functions (Eqs. 1-3) are linear. Therefore, the problem can be transformed from MINLP to MILP by modifying Eq. 6 to make the models computationally tractable. The linearized version of Eq. 6 is presented in Eq. 9.

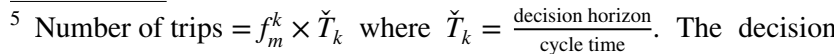
horizon in the present study is 3 -h corresponding to morning peak hour (07:00-10:00).
} 


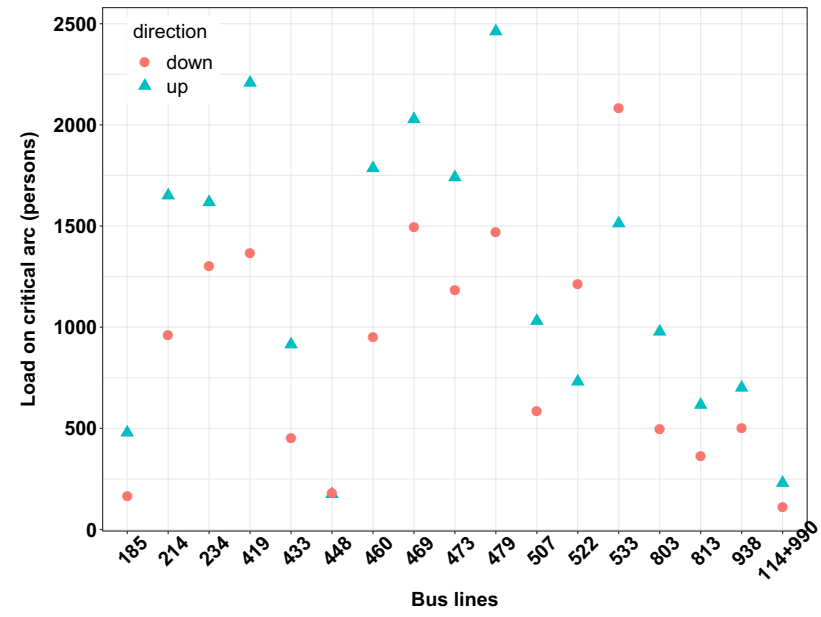

Fig. 2 Load on the critical arc of the transit routes

Table 3 Scenarios for the present case study

\begin{tabular}{|c|c|c|}
\hline Scenario & Approach & Use of bus fleet \\
\hline Scenario A & Current & Existing (i.e., Business as usual) \\
\hline Scenario B & Pragmatic & Existing +84 inter-state \\
\hline Scenario C & Pragmatic & Existing +84 inter-state +64 tourist \\
\hline Scenario D & Ideal & $\begin{array}{l}\text { Existing }+ \text { no. of inter-state buses required } \\
\text { to serve all }\end{array}$ \\
\hline Scenario E & Ideal & $\begin{array}{l}\text { Existing }+ \text { no. of tourist buses required to } \\
\text { serve all }\end{array}$ \\
\hline
\end{tabular}

The resulting models are solved in CPLEX solver 12.9.0 on an Intel ${ }^{\circledR}$ Core $^{\mathrm{TM}}$ i7 4600 CPU, 16 GB RAM, 2.70-GHz system. The CPLEX solver yielded the results within 5 minutes for 155 of the total 165 experiments $^{6}$ that are performed in this work. For the remaining ten scenarios as well, CPLEX yielded the results within 5 min after relaxing the Eq. 5 by $0.3 \%$, thus confirming that the developed models are indeed computationally tractable for real life scenarios. "Case Study: Delhi, India" has more details about the scenarios and sub-scenarios.

\section{Case Study: Delhi, India}

The proposed methodology is applied to bus service in Delhi, India. Electronic ticketing data is procured from the Delhi Integrated Multi Modal Transit System Ltd. (DIMTS), cleaned and effectively used for 17 transit lines (i.e., 34 transit routes; see $x$-axis label of Fig. 2). The ticketing data is

\footnotetext{
${ }^{6}$ Total experiments $=15$ sub scenarios $\times[3$-scenarios $(\mathrm{A}, \mathrm{B}, \mathrm{C}) \times$ 3-objective functions +2 scenarios $(\mathrm{D}, \mathrm{E})]$.
}

available for one month, however, for the present work, the average daily demand is computed using demand for the 22 working days excluding weekends. Travel demand between each pair of transit stations for the morning peak, evening peak and afternoon off-peak hours, and travel time is estimated using this ticketing data (Suman and Bolia 2019). However, for the present study, only morning peak hour travel demand is used. Figure 2 demonstrates the different levels of the load on the critical arc of each transit route. In total, as per the current operational approach, 30 low-floor and 178 semi low-floor buses are available for these 34 transit routes. ${ }^{7}$ The capacities of the buses are shown in Table 1 .

A scenario is set up for three different values of physical distancing (see "Bus Fleet and Capacities") and five different values of remaining demand multipliers (see "Reducing the Travel Demand"), i.e., total 15 cases (also called sub-scenarios).

Further, five ifferent scenarios are considered and are explained in Table 3. In scenarios B and C, the number of inter-state and tourist buses are calculated by linearly interpolating the total inter-state and tourist bus permits (see "Bus Fleet and Capacities") with respect to the total bus routes running in Delhi. In scenarios D and E, an attempt is made to identify the number of additional buses required in an ideal condition so that all the passengers can be served using the different levels of the mandated physical distancing.

\section{Results}

The fleet size for each transit route varies across the used model, scenarios and sub-scenarios (see "Case Study: Delhi, India"). The results of scenario A are shown in Fig. 3. The rows present the values of the three Key Performance Indicators (KPIs) used, viz., the proportions of passenger hours, demand and number of routes where the required physical distancing is maintained and their optimal values estimated using the objective functions (Eq. 1), objective function (Eq. 2) and objective function (Eq. 3) respectively while satisfying the constraints (Eqs. 4-9). The peach color bar shows the values of the KPIs for the current allocation, whereas the others three bars (green, sky-blue, violet) represent the optimal values of KPIs as determined by the models developed in this paper. The least demand corresponds to the first set of bars in each plot, whereas the maximum supply (i.e., available capacity) to the least value of physical distancing (first

\footnotetext{
7 The information about the distribution of the low-floor and semi low-floor buses on the 34 transit routes is unavailable. Therefore, for current allocation, this work assumes that both types of buses are distributed across the routes in their proportions, i.e., $14.4 \%$ (30 of 208) low-floor and $85.6 \%$ (178 of 208) semi low-floor buses on each route.
} 


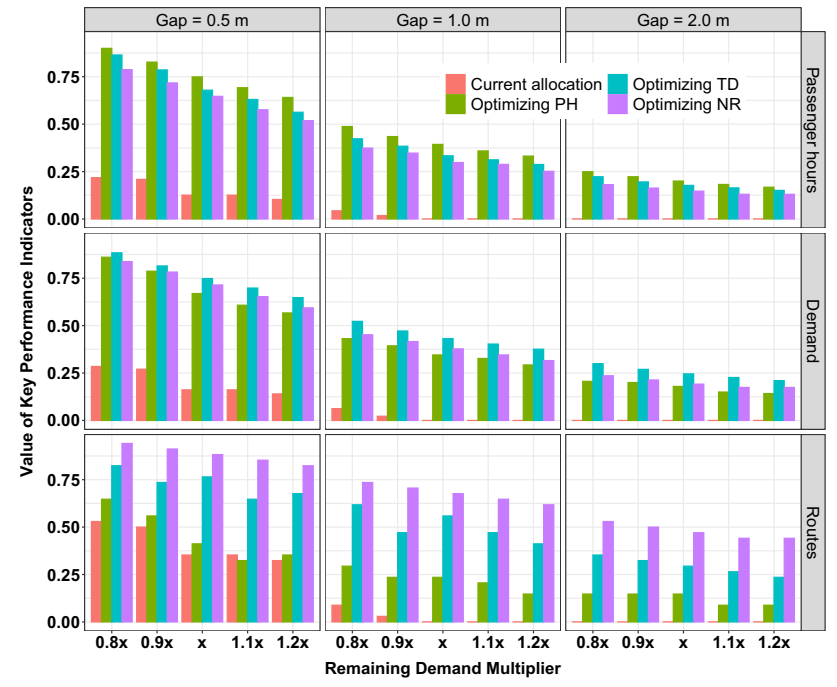

Fig. 3 Results for scenario A, where ' $x$ ' is the factor derived in "Reducing the travel demand". See Eqs. 1-3 for the models

column). As expected, the values of KPIs decrease with an increase in travel demand (from left to right) for all cases.

It is apparent from Fig. 3 that the current allocation results in abysmal levels of service corresponding to each of the three KPIs. In fact, the optimal KPIs for the current allocation and every sub-scenario of $2 \mathrm{~m}$ (as well as several sub-scenarios even for $1 \mathrm{~m}$ ) physical distancing is exactly 0 ! Moreover, the proposed optimization models efficiently reallocate buses for each sub-scenario, and a significant improvement in KPIs with respect to current allocation can be observed. Maximizing the TD (Eq. 2) reallocates the bus fleet on the routes, which may serve shorter trips as compared to maximizing $\mathrm{PH}$ and, therefore, a drop in the passenger hours can be observed (see green and sky-blue bars in the top row).

Figure 3 reveals that optimizing NR maximizes the number of routes with lesser travel demand as compared to maximizing TD (see sky-blue and violet bars in the bottom row). Therefore, optimizing NR will be useful when the requirement is to operate the maximum possible number of routes in the network.

Further, Fig. 4 shows the average travel times for scenario A while optimizing PH and TD. It is observed that as the travel demand increases, optimization of $\mathrm{PH}$ serves the passenger with longer travel times and vice versa for optimization of TD. The difference in the average trip time increases with an increase in travel demand and an increase in physical distancing. The difference becomes zero only when all the demand is met for a given level of social distancing. Therefore, if all demand cannot be served even after optimal reallocation of buses, maximizing PH should be preferred over maximizing TD in times such as the post-lockdown period, because for shorter distances, active transport is more likely to have better physical distancing. Clearly, this doesn't mean

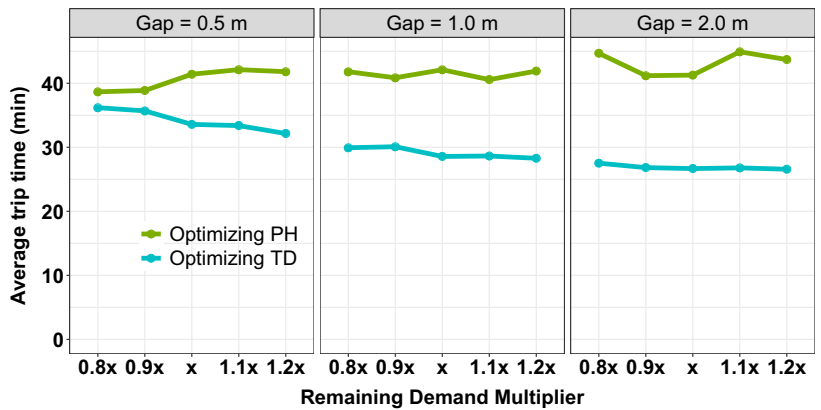

Fig. 4 Average travel time (in min) for scenario A while optimizing passenger hours (PH) and travel demand (TD)

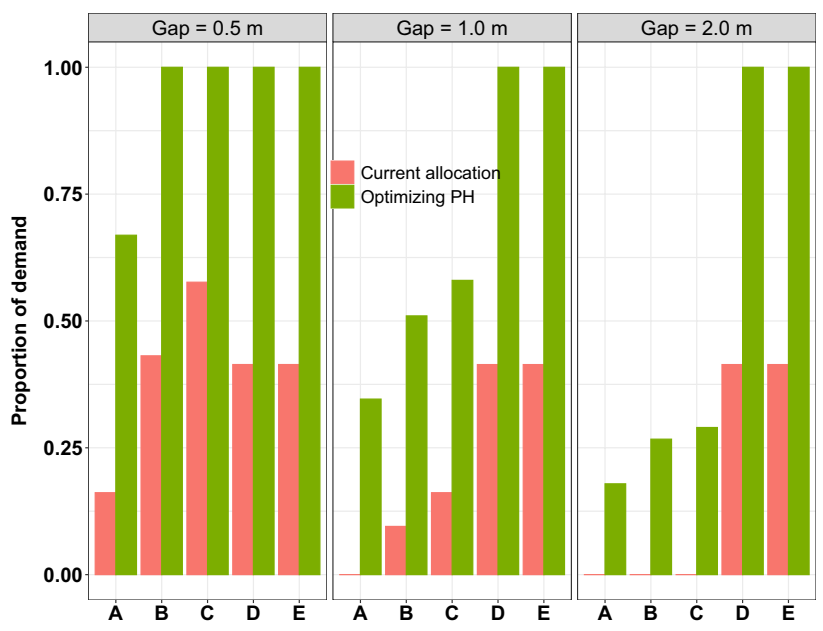

Fig. 5 Comparison of all scenarios for remaining demand multiplier $\times$

that maximizing PH neglects all shorter trips but allocates buses to routes which serves more number of longer trips as compared to those where buses are not reallocated.

In scenarios B and $\mathrm{C}$, in addition to the existing fleet of DIMTS buses, inter-state and tourist buses are also available, which clearly improves the values of KPIs for all cases shown in Fig. 3, but the trends are similar to scenario A. Therefore, the detailed results for scenarios $\mathrm{B}$ and $\mathrm{C}$ are omitted here and the summarized results for all the five scenarios are presented in Fig. 5. Due to space limitations, only the comparison between current allocation and optimizing $\mathrm{PH}$ is presented. Clearly, the current allocation approach is not able to cater to the travel demand even by including the additional available bus fleet. For example, if buses are used according to the current allocation, i.e., without using the proposed optimization models, none of the scenarios caters to even $60 \%$ of the derived travel demand for any level of distancing. However, optimum reallocation using the proposed models leads to a significant improvement in all the KPIs. Figure 5 clearly demonstrates that the operational 
Table 4 Requirement of bus fleets for Scenario $\mathrm{C}$ in the order (interstate, tourist)

\begin{tabular}{|c|c|c|c|c|c|}
\hline \multirow[t]{2}{*}{$\operatorname{Gap}(\mathrm{m})$} & \multicolumn{5}{|c|}{ Remaining demand multiplier } \\
\hline & $0.8 \times$ & $0.9 \times$ & $1 \times$ & $1.1 \times$ & $1.2 \times$ \\
\hline 0.5 & $(22,0)$ & $(44,0)$ & $(67,0)$ & $(84,12)$ & $(84,50)$ \\
\hline 1 & \multicolumn{5}{|c|}{$\begin{array}{l}\text { Need more than }(84,64) \text { additional buses to meet the } \\
\text { reduced demand, even after optimization }\end{array}$} \\
\hline 2 & \multicolumn{5}{|c|}{$\begin{array}{l}\text { Need more than }(84,64) \text { additional buses to meet the } \\
\text { reduced demand, even after optimization }\end{array}$} \\
\hline
\end{tabular}

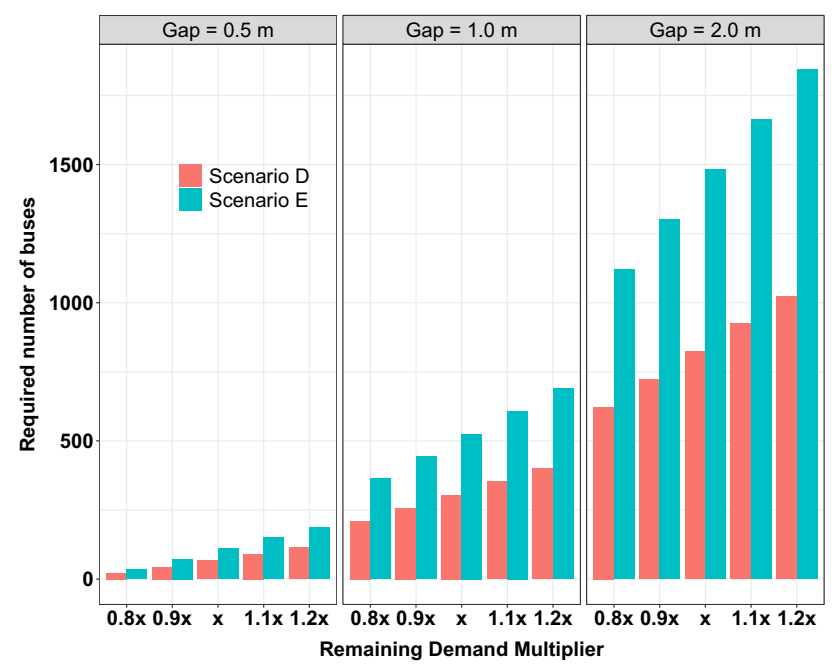

Fig. 6 Requirement of bus fleets for scenarios D and E

approach implied by the optimization model is far better than the approach currently being followed for every scenario.

Figure 6 and Table 4 reveal that if buses in scenarios B and $\mathrm{C}$ are reallocated using the optimization models, then these buses are sufficient to provide a physical distancing of $0.5 \mathrm{~m}$, even in the maximum possible travel demand subscenario, i.e., for remaining demand multiplier $1.2 \times$. Further, when the physical distancing required is $2 \mathrm{~m}$, even the additional buses of scenario $\mathrm{C}$ are insufficient to meet the minimum estimated travel demand (i.e., $0.8 \times$ ) if the current allocation strategy is used. However, with the proposed models, $45 \%$ of the public bus travel demand can be met while maintaining a distancing of $2 \mathrm{~m}$.

Furthermore, a comparison of scenarios D and $\mathrm{E}$ is shown in Fig. 6. The optimum number of the required inter-state (scenario D) or tourist (scenario E) buses increases with an increase in demand as well as with an increase in physical social distancing. While, this trend is trivially expected, the numbers given by the models are not trivial, they represent a useful policy parameter: the number of buses required for different levels of distancing.

Overall, the results clearly reveal that restarting postlockdown public transport operations, while maintaining the required distancing, is next to impossible with the current allocation based on BAU approach. On the other hand, the proposed optimization models not only make it feasible to safely begin plying buses again, they result in significantly better KPIs across all scenarios of demand and additional resource deployment.

\section{Conclusions and Recommendations}

The present study provides a policy prescription to the public transport-specifically bus-operators in the immediate aftermath of lockdown, a situation where travel demand is uncertain, and ample physical distancing is mandatory. It is shown that there is a clear gap between the derived demand (see Remaining Demand Multiplier, "Reducing the Travel Demand") and supply. This gap can be narrowed significantly by using the optimization models proposed in this work. These models do not necessarily and exclusively rely on capital infusion and heavy expenditure, instead on efficiently allocation of whatever number of buses is available on the routes. Further, even after optimization, an additional bus fleet is required to fully satisfy the entire demand in all scenarios. To sum up, the work reveals the following key insights for decision makers:

- The travel demand in none of the routes can be served in the Business-as-usual (BAU) approach, i.e., using the current operational strategy (allocation) and no additional buses, at any level of derived travel demand and physical distancing.

- The operational strategy of reallocation using the proposed models significantly improves all KPIs in every scenario, including all levels of travel demand and physical distancing.

- The requirement of additional buses can be satisfied with idling inter-state and tourist buses with KPIs that depend on the travel demand, and the required distancing level.

- The analysis can determine the minimum number of buses, along with the corresponding operational strategy, to be added to serve $100 \%$ of the travel demand, should the decision makers choose that to be the policy mandate.

Though the optimization models are applied to a case study of Delhi, India, they can be applied to any other city provided the requisite data is available.

For effective operations, we conclude with some practical remarks to supplement our model insights. It would help to determine the travel demand in advance by asking the travelers to enter their travel information (e.g., origin, destination, departure time, etc.) on the widely used "Arrogya Setu" mobile application for a typical day. In order to avoid any privacy concerns, the origin destination information can 
be entered in terms of nearby landmarks (see Vardhan et al. 2020 for a use-case of the possible feature) and no personal information should be asked or tracked. For the unplanned/ instant trips, the real-time arrival as well as occupancy information of the transit modes can be made available so that unregistered travelers can plan their trip accordingly. The registered travelers can purchase their ticket on the app to reduce their contact with conductors. The rest of the travelers can be encouraged/mandated to pay using UPI/QR code in the buses which will decrease the necessity of conductors in the buses. Further, boarding/alighting permissions can be limited to the rear gate alone to reduce the risk to the driver. Practically, physical distancing can be maintained well, if the seating, as well as the standing spaces, are clearly marked in the buses. It is recommended to sanitize the vehicles before the first trip and after every trip which is likely to increase the terminal time (time spent at either of the terminals for sanitation) of the transit vehicles. For the operations, the guidelines by the competent authorities shall be followed all times.

Acknowledgements The authors wish to thank anonymous reviewers for their valuable comments. The responsibility of any remaining errors stays with the authors.

Author Contributions Conceptualization: AA, NBB; research design: HKS, NBB, AA; model formulation and running experiments: HKS; formal analysis and investigation: HKS, AA; manuscript preparation: AA, HKS. All authors read and approved the final manuscript.

\section{References}

Agrawal K, Suman HK, Bolia NB (2020) Frequency optimization models for reducing overcrowding discomfort. Transp Res Rec. https ://doi.org/10.1177/0361198120912230
CRRI (2020) Guidelines for public transport and feeder modes considering social distancing norms. Technical report, Central Road Research Institute

Fang H, Wang L, Yang Y (2020) Human mobility restrictions and the spread of the novel coronavirus (2019-nCoV) in China. Tech Rep Natl Bur Econ Res. https://doi.org/10.1101/2020.03.24.20042424

Govt. of NCT of Delhi (2020) Transport department: bus permit dataset. http://tte.delhigovt.nic.in/wps/wcm/connect/doit_transport/ Transport/Home/Permits/. Accessed 19 Apr 2020

Müller SA, Balmer M, Neumann A, Nagel K (2020) Mobility traces and spreading of COVID-19. Technische Universität, Berlin. https ://doi.org/10.14279/depositonce-9835

Rangwala L, Mathews R, Sridhar S (2014) Shifting discourse about transit-oriented development in Mumbai, India. Transp Res Rec J Transp Res Board 2451(1):60-67. https://doi.org/10.3141/2451-07

Shi P, Keskinocak P, Swann JL, Lee BY (2010) The impact of mass gatherings and holiday traveling on the course of an influenza pandemic: a computational model. BMC Public Health. https:// doi.org/10.1186/1471-2458-10-778

Suman HK, Bolia NB (2019) Mitigation of overcrowding in buses through bus planning. Public Transp 11:159-187. https://doi. org/10.1007/s12469-019-00197-x

Tiwari G, Jain D, Rao KR (2016) Impact of public transport and non-motorized transport infrastructure on travel mode shares, energy, emissions and safety: case of Indian cities. Transp Res Part D Transp Environ 44:277-291. https://doi.org/10.1016/j. $\operatorname{trd} .2015 .11 .004$

Vardhan H, Rai I, Kathait N, Agarwal A (2020) Crowd-sourced web survey for household travel diaries. Working paper, Indian Institute of Technology Roorkee. See WP\#30 at http://faculty.iitr. ac.in/ amitfce/publications.html. Accessed 1 May 2020

Vuchic VR (2004) Urban transit: operations planning and economics. Willey, Hoboken

WHO (2020) Novel Coronavirus (2019-nCoV) situation report-10: technical report. World Health Organization, Washington

WHO (2020) Novel Coronavirus (2019-nCoV) situation report-94: technical report. World Health Organization, Washington

Publisher's Note Springer Nature remains neutral with regard to jurisdictional claims in published maps and institutional affiliations. 